\title{
Interrelation of the effects of binocular disparity and perspective cues on judgments of depth and height
}

\author{
H. C. van der MEER \\ University of Nijmegen, Nijmegen, The Netherlands
}

\begin{abstract}
The effects of binocular disparity (aniseikonia) and perspective cues operating together on judgments of depth and height were studied, both when these stimulus variables operated in the same direction and when they were in conflict. Both depth cues were effective upon the perception of depth and height. The effects of binocular disparity and perspective cues upon perceived depth were found to be additive. The effects of these depth cues upon perceived height showed some interaction in the sense that, operating together, the effect of the perspective cue was stronger than the separate effect of the perspective cue, both when binocular disparity and perspective cues operated in the same direction and when they were in conflict. This interactive effect increased with increasing strength of the perspective cues. The size-distance invariance hypothesis was confirmed under the present experimental conditions. By a causal analysis of inference, this invariant relation could be explained in the following way: both the perceived depth and the perceived height of the sides of the patterns were directly determined by binocular disparity and perspective cues, but the perceived height was also indirectly determined through change of perceived depth. A direct causal relation between perceived depth and perceived height was found.
\end{abstract}

Harker (1958) found that when binocular disparity was supplemented with monocular cues to depth, observers had better stereoacuity than when the binocular cues alone were present. Harker suggested that this could be attributed to the fact that binocular and monocular cues provide the observer with independent information about the depth interval between two objects. The observers integrated the monocular and binocular stimulation in a supplemental manner. Jameson and Hurvich (1959) concluded, in an experiment on the relation between stereoscopic acuity and observation distance, that when multiple stimulus factors are permitted to vary naturally with changes in object distance, all of these multiple factors seem to contribute cooperatively and in a simple summative fashion to the observer's perceptual sensitivity to distance changes. The monocular cues in neither experiment were fully identified.

In the present study, we will investigate the way in which binocular disparity and perspective cues affect the perception of depth, both when these cues operate in the same direction and when they are in conflict. As cues to the perception of depth or distance are also implicated in size perception, we will simultaneously study the effects of binocular disparity and perspective cues on the perception of size.

There are a number of papers on the effect of combining monocular depth cues with binocular disparity upon perceived depth. Very few studies have simultaneously investigated their effects on both perceived depth and perceived size. Most studies on perceived depth have concentrated upon the effects of cue conflict and upon the relative strengths of the cues. These cue-conflict studies have not always been consistent in outcome. Schriever (1925) found that pseudoscopic viewing of perspective scenes produces an impression of depth that is consistent with perspective and not with disparity. This study was only qualitative. In Schriever's photographs, more monocular depth cues than perspective may have been effective. Gillam (1968) found that when perspective and stereopsis (aniseikonia) were in conflict, the perceived depth was reduced. Aniseikonia predominated perspective. The same was found by Van der Meer (Note 1) in combinations of vertical aniseikonia or the induced effect and perspective. In contrast to these latter two studies, Epstein and Morgan-Paap (1974) found that perspective fully dominated binocular disparity (aniseikonia). These authors concluded that the effectiveness of the perspectival input was in no way modified by conflicting stereoscopic slant depth. Their stimulus figures were photographic negatives of rotated rectangles, so that these figures had two monocular depth cues: perspective and foreshortening. The perceived rotation in the monocular condition approximated the real rotation of the rectangles. The monocular cues, perspective and foreshortening, apparently delivered sufficient information to reliably estimate the angle of rotation. Conflicting binocular disparity was not effective under these circumstances.

The relationship between perceived size and per- 
ceived distance can be described by the size-distance invariance hypothesis, which states that, for a given visual angle, there is a unique ratio between perceived size and perceived distance.

The size-distance invariant relationship can be explained in at least three ways: (1) The perceived depth is determined by stimulus cues and the perceived height is proportionally related to this perceived depth. (2) The perceived height is determined by stimulus cues and the perceived depth is proportionally related to this perceived height. (3) Both perceived depth and perceived height are determined by the same stimulus cues in the same way, and, consequently, they are correlated with each other.

Epstein and Landauer (1969) and Landauer and Epstein (1969) suggested that, under certain circumstances, judged size and judged distance were independent consequences of the same stimulus variable, namely visual angle. This is in agreement with case 3 . Gogel (1971) considered it unlikely that visual angle would independently determine perceived size and perceived distance. A causal analysis of inference, developed by Blalock (1962) and Simon (1954), can be applied to determine which of these explanations is valid, when perceived depth and perceived size are simultaneously obtained under various combinations of stimulus cues. Oyama (1974) applied this type of analysis and found that perceived size and perceived distance were directly determined by visual angle and by convergence. The observed relation between perceived size and perceived distance was not based on a direct causal relation between them. Mori and Watase (cited by Oyama, 1977), on the contrary, found for the stimulus variables, visual angle and physical distance, a causal relation between perceived size and perceived distance for most of their subjects.

In the present study, the questions to be resolved were the following: (1) What kind of interrelation have binocular disparity and perspective cues in their effects on perceived depth and perceived size? (2) Does the size-distance invariance hypothesis hold under the conditions of this experiment? (3) Do binocular disparity and perspective cues determine perceived depth and perceived size under the conditions of this experiment in the same way? To resolve this question we shall apply a causal analysis of inference in using partial correlations (Blalock, 1962; Simon, 1954).

\section{METHOD}

\section{Apparatus}

The patterns were vertical square-wave gratings with a linear perspective. Figure 1 shows one of the patterns. They were generated by computer and projected by two Kodak Carousel projectors on a metallic screen. By means of an arrangement of polarizing filters and glasses, the monocular patterns were projected with the centers in register, so that each eye could see only one of the monocular patterns.

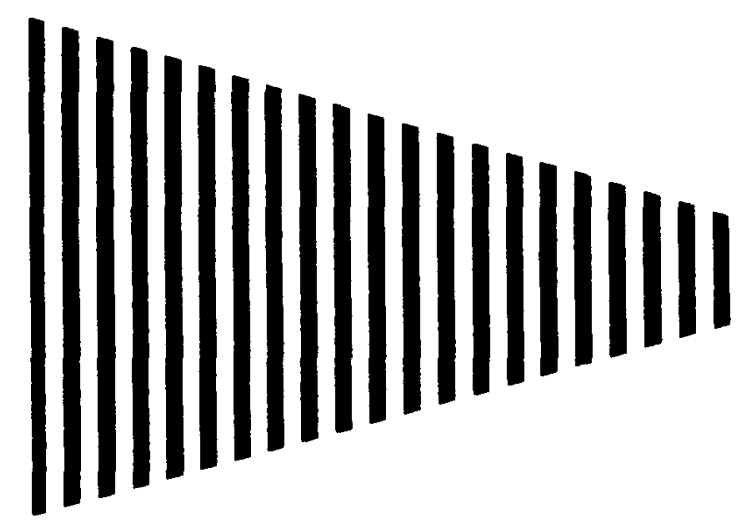

Figure 1. An example of the patterns used.

The height of the left side of all patterns was $15.0 \mathrm{~cm}$. The height of the right side of the patterns was variable, namely $15.0 \mathrm{~cm}$ (without perspective cue), $12.0,8.3,5.0$, and $1.7 \mathrm{~cm}$.

The spatial frequency of the projected gratings, defined as the number of cycles per degree of visual angle, was 5 cycles/deg. The pattern consisted of 20.5 cycles. The contrast of the gratings was .70 , the overall luminance $18 \mathrm{fL}$. The observation distance was $300 \mathrm{~cm}$. A spatial frequency of 5 cycles $/ \mathrm{deg}$ was chosen, because Van der Meer (1978) found that this was the lowest spatial frequency (and thus the largest pattern) with a maximum of perceived rotation in aniseikonia.

For the stereoscopic or aniseikonic session, the five patterns were paired with a pattern that was magnified $9.8 \%$ in the horizontal direction. The heights of the left and right sides of the monocular patterns of each stereo pair were equal. When the magnified pattern was seen by the right eye, aniseikonia and perspective were in agreement; when the magnified pattern was seen by the left eye, they were in conflict. In the perspective session, the four patterns with a perspective cue were used.

The head of the observer was fixed by means of a head and chin support. The observer was free to move his eyes.

\section{Subjects}

The subjects were undergraduates who were fulfilling a course requirement. They were first tested for normal visual acuity and normal stereoacuity. They had to pass a score of 9 on the Titmus stereo test. Then they were tested with aniseikonic random-dot patterns and subsequently had a 15 -min training period with these patterns. When a subject perceived rotation in the expected direction, he was admitted to the experiment. Twenty-five subjects were tested; seven were rejected, four because of subnormal stereoacuity and three for not perceiving rotation in the aniseikonic random-dot patterns.

\section{Procedure}

There were two experimental sessions: a perspective session and an aniseikonic session. Half of the subjects started with the perspective session and the other half with the aniseikonic session. In the perspective session, the subject looked binocularly to the patterns of a spatial frequency of 5 cycles/deg. In this session, four patterns with different strengths of perspective were used. Each pattern was presented four times. The 16 presentations were shown in a random sequence. The subject had to estimate the degree of perceived rotation and the height of the left and right side of each pattern.

In the aniseikonic session, the five pairs of patterns were presented. The magnified monocular patterns were presented to the right eye (agreement between aniseikonia and perspective) as well as to the left eye (conflict between aniseikonia and perspective). The resulting 10 trials were presented in a random sequence. After each series of 10 trials, there was a rest of 15 min. Each 
series was presented four times in a different random sequence. Between trials, the subject had to close his eyes, while the experimenter adjusted the patterns and the polarizing filters before the projectors.

\section{Judgment of Rotation}

A screen $(30 \times 30 \mathrm{~cm})$, made of white cardboard and rotating about a vertical axis, was located just below the projected pattern. A vertical black line down the center and three horizontal black lines were drawn on the white cardboard. The subject indicated the apparent rotation of the pattern by adjusting this screen to appeat parallel to the pattern. The subject could adjust this rotating screen by pulling a string attached to its vertical borders. The experimenter could read the angle of rotation in degrees at the rear of the rotating screen.

The subjects were instructed to be very careful to adjust the screen to the rotation of the whole pattern and not to adjust the upper size of the screen parallel to the lower side of the pattern.

\section{Judgment of Height}

After the subject had adjusted the rotating screen to the perceived rotation of the pattern, he was asked to estimate the height of the left and right sides of the apparently rotated pattern. To facilitate the estimation of the height of the sides of the patterns, a screen with 16 bars of different heights was presented on the right of the projected pattern. These bars, of the same width as those of the projected pattern, had heights of $1,2,3$, $\ldots, 15$, and $16 \mathrm{~cm}$, respectively. They were presented in two rows of 8 bars. The subjects were instructed to match the perceived height of the sides of the patterns with the bars on the screen on the right. For instance, if a subject judged the height of a side of the apparently rotated pattern to be between the height of the $7-\mathrm{cm}$ bar and the height of the $8-\mathrm{cm}$ bar on the screen at the right, he knew that the perceived height of the side of the pattern was at least $7 \mathrm{~cm}$. He had to estimate the difference in millimeters.

\section{RESULTS}

\section{Perceived Rotation}

The mean perceived rotations of the 18 subjects are presented in Table 1. The degree of perspective of the pattern is expressed by means of the height of the right side of the patterns. The mean rotations for when the direction of rotation for aniseikonia and perspective was in the same direction are presented in the first column of the table. From these data, it is clear that the perceived rotation increases when the perspective cue becomes stronger. The monotonic increasing trend is very significant $(z=10.334)$.

When the aniseikonic and perspective cues are in conflict, column 2 of Table $1,{ }^{1}$ there is a very sig- nificant monotonic decreasing trend $(z=9.757)$. For 12 of the 18 subjects, stereopsis dominates over perspective, for 4 subjects perspective dominates over stereopsis, and for 2 subjects perspective dominates only in the strongest two perspective conditions.

The means of the perceived rotations in the perspective conditions are presented in column 3 . This trend is also very significant $(z=7.446)$.

From the data, it is clear that both stereopsis and perspective cues have an effect on the perceived rotation of the patterns. To answer the question about the interrelation of the effects of binocular disparity and perspective cues upon perceived depth, the perceived rotations in the perspective condition are subtracted from the mean rotations when aniseikonia and perspective agree in direction (column 4), and added to the perceived rotations when they are in conflict (column 5). In column 4, the four values are approximately equal to the perceived rotation in the aniseikonic condition without perspective of $37.5 \mathrm{deg}$ (column 1). The standard error is $4.7 \mathrm{deg}$, so the differences are not significant. The values in column 5 are not significantly different from the value of $42.2 \mathrm{deg}$ in column 2 , aniseikonia without perspective. The standard error is $5.2 \mathrm{deg}$.

For the group means, the effects of binocular disparity (aniseikonia) and perspective cues on the perceived rotation of the patterns are additive and do not interact.

In the left half of Table 2, the sum of the values of the perceived rotations in the aniseikonic condition (A) and in the perspective condition (P) are subtracted from the values of the perceived rotation when aniseikonia and perspective cues operate in the same direction $(\overline{\mathrm{A}+\mathrm{P}})$. In the right half of Table 2 , the difference of the values of the perceived rotation in the $\mathbf{A}$ condition and in the $\mathbf{P}$ condition are subtracted from the values of the perceived rotation when aniseikonia and perspective cues are in conflict $(\overline{A-P})$. When the effects of $A$ and $P$ are additive, the values in Table 2 will not significantly differ from zero. There is only one subject who has values that differ significantly from zero. For this subject, perspective cues had only a very small effect in the $\overline{\mathrm{A}+\mathrm{P}}$ and $\overline{\mathrm{A}-\mathrm{P}}$ conditions.

Table 1

Perceived Rotations in Degrees in the Aniseikonic and Perspective Conditions

\begin{tabular}{|c|c|c|c|c|c|}
\hline $\begin{array}{l}\text { Height } \\
\text { Right } \\
\text { Side }\end{array}$ & $\begin{array}{l}A \text { and } P \text { in } \\
\text { Agreement } \\
\text { (1) }\end{array}$ & $\begin{array}{l}A \text { and } P \text { in } \\
\text { Conflict } \\
\text { (2) }\end{array}$ & $\begin{array}{c}\mathbf{P} \\
(3)\end{array}$ & $\begin{array}{c}(1)-(3) \\
(4)\end{array}$ & $\begin{array}{c}(2)+(3) \\
(5)\end{array}$ \\
\hline 15.0 & 37.5 & 42.2 & & & \\
\hline 12.0 & 45.2 & 35.3 & 8.0 & 37.2 & 43.3 \\
\hline 8.3 & 51.3 & 29.5 & 14.3 & 37.9 & 43.8 \\
\hline 5.0 & 57.0 & 24.0 & 19.1 & 37.9 & 43.1 \\
\hline 1.7 & 60.6 & 17.8 & 23.9 & 36.7 & 41.7 \\
\hline
\end{tabular}

Note-A positive sign means rotation in the direction of binocular disparity. 
Table 2

Additivity of the Effects of Aniseikonic and Perspective Depth Cues in Case of Perceived Depth for 18 Subjects

\begin{tabular}{|c|c|c|c|c|c|c|c|c|c|c|}
\hline \multirow[b]{2}{*}{ Subject } & \multicolumn{5}{|c|}{$\overline{\mathrm{A}+\mathrm{P}}-(\mathrm{A}+\mathrm{P})$} & \multicolumn{5}{|c|}{$\overrightarrow{A-\mathbf{P}}-(A-P)$} \\
\hline & 12.0 & 8.3 & 5.0 & 1.7 & Mean & 12.0 & 8.3 & 5.0 & 1.7 & Mean \\
\hline 1 & -.9 & -2.3 & .5 & 4.5 & .20 & 2.3 & 3.1 & -.9 & -4.2 & .08 \\
\hline 2 & 1.7 & 2.2 & -.2 & -3.8 & .03 & 1.3 & -3.2 & -4.8 & -4.2 & --2.73 \\
\hline 3 & 2.3 & .8 & 2.0 & -5.3 & .75 & -.8 & 3.2 & .8 & 0.0 & .80 \\
\hline 4 & -1.3 & 3.5 & .5 & .5 & .80 & $5.8^{*}$ & 5.0 & $8.0^{*}$ & 5.0 & 5.95 \\
\hline 5 & -1.2 & -1.1 & .1 & .2 & .50 & -.3 & -1.4 & -.5 & -.3 & -.63 \\
\hline 6 & -.9 & -3.0 & 1.7 & 1.0 & .30 & .7 & 2.9 & 3.8 & .7 & 2.03 \\
\hline 7 & .7 & -1.1 & -.7 & -1.0 & .53 & 2.1 & -.3 & 4.7 & -.3 & 1.55 \\
\hline 8 & 1.8 & -2.1 & -1.0 & -.2 & .38 & -.6 & 2.9 & 1.7 & -2.4 & .40 \\
\hline 9 & .5 & -1.0 & .5 & .0 & .00 & .5 & 3.0 & 1.0 & -3.5 & .25 \\
\hline 10 & 2.0 & 2.4 & 7.4 & 3.7 & 3.88 & 3.7 & 3.7 & -5.7 & -4.6 & -.73 \\
\hline 11 & 5.0 & 1.1 & 2.6 & -2.4 & 1.58 & -1.0 & -4.4 & -5.0 & .3 & -2.53 \\
\hline 12 & -.4 & .2 & 1.0 & .8 & .40 & -1.0 & -.2 & .3 & -.5 & -.35 \\
\hline 13 & $-13.0^{*}$ & $-9.9 *$ & $-10.9 *$ & $-11.5^{*}$ & $-11.33^{*}$ & $7.0^{*}$ & $7.9 *$ & $8.9 *$ & $13.8^{*}$ & $9.40^{*}$ \\
\hline 14 & -4.1 & 4.4 & 3.6 & 1.4 & 1.08 & .3 & 2.9 & 2.5 & -1.5 & 1.05 \\
\hline 15 & 1.7 & .9 & $-\quad .7$ & -1.5 & .10 & 2.1 & -.8 & -.3 & -.3 & .18 \\
\hline 16 & 1.3 & -1.1 & .0 & .8 & .25 & -.1 & 2.9 & 1.7 & -2.4 & .53 \\
\hline 17 & .5 & .3 & 1.7 & -1.8 & .18 & -3.3 & .2 & .8 & -1.0 & -.83 \\
\hline 18 & .5 & -3.0 & .5 & 1.0 & .50 & .8 & 1.0 & -1.0 & -3.5 & -.68 \\
\hline
\end{tabular}

Note-The strength of the perspective cue is indicated by the height of the right side of the patterns.

*Significant deviations from zero beyond the $5 \%$ level.

The conclusion from this analysis is that the effects of aniseikonia and perspective cues on perceived depth are additive under the circumstances of this experiment.

\section{Perceived Height}

The estimated heights of the left and right sides of the patterns expressed in percentages of the objective heights, are presented in Table 3 . The perceived heights are dependent on the degree of perceived rotation. When aniseikonia and perspective are in agreement $(\overline{\mathrm{A}+\mathrm{P}})$, the left side of the pattern is perceived in front, and with increasing perceived rotation, the perceived height decreases, in agreement with constancy scaling. The decreasing trend is very significant $(\mathrm{z}=6.524)$.

With increasing rotation, the right side of the patterns recedes more backwards, and in agreement with

Table 3

Perceived Height in Proportion of the Objective Height of the Left and Right Sides of the Patterns

\begin{tabular}{llrrrrrr}
\hline & & \multicolumn{2}{c}{$\overline{A+P}$} & \multicolumn{2}{c}{$\overline{A-P}$} & \multicolumn{2}{c}{$P$} \\
$H$ & & Left & Right & \multicolumn{1}{c}{ Left } & Right & Left & Right \\
\hline \multirow{3}{*}{15.0} & Mean & 94.8 & 100.0 & 102.6 & 92.6 & & \\
& SE & .74 & 1.13 & 1.27 & .89 & & \\
12.0 & Mean & 93.9 & 102.0 & 101.2 & 94.2 & 98.0 & 101.3 \\
& SE & .95 & 2.97 & 1.50 & 1.07 & 1.16 & 1.33 \\
& Mean & 92.8 & 105.2 & 100.5 & 94.4 & 98.2 & 102.7 \\
8.3 & SE & .92 & 3.62 & 1.51 & 1.24 & 1.09 & 1.08 \\
& Mean & 92.7 & 108.3 & 100.0 & 95.1 & 98.2 & 102.3 \\
5.0 & SE & 1.01 & 3.13 & 1.50 & 1.51 & 1.15 & 1.87 \\
& Mean & 91.9 & 111.9 & 98.9 & 96.6 & 98.0 & 103.2 \\
1.7 & SE & 1.07 & 2.52 & 1.62 & 2.81 & .90 & 2.56 \\
\hline
\end{tabular}

Note $-H=$ height of right side. constancy scaling the perceived height increases. This trend is very significant $(\mathrm{z}=8.487)$.

When aniseikonia and perspective cues are in conflict $(\overline{\mathrm{A}-\mathrm{P}})$, the left side of the pattern is perceived behind the surface of the projection screen if aniseikonia dominates and in front of it if perspective dominates. In both cases, the perceived height of the left side decreases with increasing perspective. This trend is very significant $(z=6.755)$.

The right side of the pattern is perceived as being in front of the surface of the projection screen if aniseikonia dominates and behind it if perspective dominates. In both cases, the perceived height of the right side increases with increasing perspective. This trend is also very significant $(\mathrm{z}=6.986)$.

In the perspective condition, there is no difference in the perceived height of the left side of the patterns. The differences in height estimates of the right side of the patterns are not significant $[F(3,51)=$ .456]. There is also no significant trend in the height estimates of the right side $(z=1.521)$. There is, however, a significant difference in height estimates between the left and the right sides of the patterns. Tested with the two-sided sign test, this difference is significant at the .01 level. The right, receding sides of the patterns are perceived as being relatively higher than the left sides.

To study the interrelation of the effects of aniseikonia and perspective cues on perceived height, the values of $\overline{A+P}-(A+P)$ for the right side and of $\overline{\mathrm{A}-\mathrm{P}}-(\mathrm{A}-\mathrm{P})$ for the left side of the patterns are shown for all 18 subjects in Table 4 . For additivity to hold, these values must not differ significantly from zero. Most subjects have at least some $\overline{\mathbf{A}+\mathbf{P}}$ $(A+P)$ values which deviate significantly from zero. 
Table 4

Values of $\overline{\mathrm{A}+\mathrm{P}}-(\mathrm{A}+\mathrm{P})$ and of $\overline{\mathrm{A}-\mathrm{P}}-(\mathrm{A}-\mathrm{P})$ for the Perceived Height of the Patterns for 18 Subjects

\begin{tabular}{|c|c|c|c|c|c|c|c|c|c|c|}
\hline \multirow[b]{2}{*}{ Subject } & \multicolumn{5}{|c|}{$\overline{A+P}-(A+P)($ Right Side $)$} & \multicolumn{5}{|c|}{$\overline{A-P}-(A-P)($ Left Side $)$} \\
\hline & 12.0 & 8.3 & 5.0 & 1.7 & Mean & 12.0 & 8.3 & 5.0 & 1.7 & Mean \\
\hline 1 & $10.1^{*}$ & -2.0 & $12.0^{*}$ & -7.7 & 3.10 & $-12.5 *$ & $-12.5 *$ & $-9.8^{*}$ & $-15.0^{*}$ & $-12.45^{*}$ \\
\hline 2 & -7.6 & -.1 & -1.7 & $11.2 *$ & .45 & $13.5^{*}$ & $11.8^{*}$ & $10.1 *$ & $8.4 *$ & $10.95 *$ \\
\hline 3 & -3.5 & $7.7 *$ & $7.1^{*}$ & $9.7 *$ & $5.25^{*}$ & $-4.6^{*}$ & $-4.5^{*}$ & $-8.2 *$ & $-5.6^{*}$ & $-5.73 *$ \\
\hline 4 & -2.4 & -3.3 & $9.9 *$ & $18.6^{*}$ & $5.70^{*}$ & .8 & 5.3 & -5.0 & $-11.8 *$ & -2.68 \\
\hline 5 & .6 & $2.7^{*}$ & $7.0^{*}$ & $12.2 *$ & $5.63^{*}$ & $-1.8^{*}$ & -1.2 & $-2.0^{*}$ & $-2.3 *$ & $-1.83^{*}$ \\
\hline 6 & .9 & .5 & $5.7 *$ & $3.6^{*}$ & $2.68^{*}$ & -.3 & -.6 & $-4.4 *$ & $-2.5^{*}$ & $-1.95^{*}$ \\
\hline 7 & .4 & $5.4^{*}$ & $8.1^{*}$ & $13.8 *$ & $6.93 *$ & -.6 & $-2.6^{*}$ & $-3.3 *$ & $-4.1 *$ & $-2.65^{*}$ \\
\hline 8 & .1 & $2.6^{*}$ & $6.5^{*}$ & $11.4 *$ & $5.15^{*}$ & .4 & $-2.0^{*}$ & $-\quad .9$ & -1.0 & $-\quad .88$ \\
\hline 9 & 2.5 & $3.4 *$ & $4.4^{*}$ & $5.4 *$ & $3.93^{*}$ & -.6 & -1.4 & $-2.0^{*}$ & -1.5 & $-1.38^{*}$ \\
\hline 10 & $12.3^{*}$ & 4.3 & $4.7^{*}$ & $8.2^{*}$ & $7.63^{*}$ & $-14.4^{*}$ & $-20.9^{*}$ & $-23.6^{*}$ & $-24.0^{*}$ & $-20.73^{*}$ \\
\hline 11 & $4.0^{*}$ & $4.7^{*}$ & $6.9 *$ & $13.8 *$ & $7.35 *$ & $-14.7 *$ & $-12.0^{*}$ & $-12.4 *$ & $-15.5^{*}$ & $-13.55^{*}$ \\
\hline 12 & $-5.3 *$ & -2.3 & -1.9 & $-5.2 *$ & $-3.68^{*}$ & $11.1^{*}$ & $8.4^{*}$ & $7.8 *$ & $15.4^{*}$ & $10.68 *$ \\
\hline 13 & .3 & .9 & 3.1 * & $4.6^{*}$ & $2.23^{*}$ & $-\quad .1$ & 5.0 & -5.0 & $-15.2 *$ & -3.83 \\
\hline 14 & .6 & $3.1^{*}$ & $7.1^{*}$ & $12.3^{*}$ & $5.78 *$ & -1.0 & $-\quad .8$ & $-2.0^{*}$ & $-3.4^{*}$ & $-1.80^{*}$ \\
\hline 15 & -.4 & $3.4 *$ & $8.1^{*}$ & $11.8 *$ & $5.73^{*}$ & .2 & $-2.6^{*}$ & $-3.3^{*}$ & $-5.4^{*}$ & $-2.78^{*}$ \\
\hline 16 & .1 & $3.6^{*}$ & $6.5^{*}$ & $11.4^{*}$ & $5.40^{*}$ & $-\quad .4$ & $-2.0^{*}$ & $-\quad .9$ & $-2.1^{*}$ & $-1.35^{*}$ \\
\hline 17 & -3.0 & $7.7^{*}$ & $7.1 *$ & $11.7^{*}$ & $5.88^{*}$ & $-5.0^{*}$ & $-4.5^{*}$ & $-8.2 *$ & $-5.6^{*}$ & $-5.83 *$ \\
\hline 18 & 2.5 & $3.4^{*}$ & $6.4^{*}$ & $10.1^{*}$ & $5.60^{*}$ & $-\quad .6$ & -1.4 & $-4.0 *$ & $-4.2^{*}$ & $-2.55^{*}$ \\
\hline
\end{tabular}

*Significant deviations from zero beyond the $5 \%$ level

Note-The perspective cue is indicated by the height of the right side of the patterns.

With the exception of one subject, the mean value for each subject is positive. This means that when aniseikonic and perspective cues operate in the same direction, there is an interactive effect in the sense that the combined effect is stronger than the sum of the separate effects of aniseikonia and perspective cues on perceived height. A test for monotonic trend was used to see if this interactive effect increased with increasing strength of the perspective cue. It was found that this trend was very significant $(z=6.165)$.

In the right half of Table 4 , the values $\overline{\mathrm{A}-\mathrm{P}}-$ (A - P) (aniseikonia and perspective cues in conflict) are shown. The significant deviations from zero are marked in Table 4. Sixteen subjects have a mean negative value, and two subjects have a mean positive value of $\overline{\mathrm{A}-\mathrm{P}}-(\mathrm{A}-\mathrm{P})$. For Subjects 1, 2, 3, 10, 11 , and 17, perspective dominated over aniseikonia. The positive value of Subject 2 is due to misperception, since, in the perspective condition, the height of both the left side and the right side of the patterns were strongly overestimated. Subject 12 suppressed the influence of the perspective cue when $A$ and $P$ operated together. A negative value of $\overline{A-P}$ $(A-P)$ means that the difference of the isolated effects of $A$ and $P$ on perceived height is larger than when $A$ and $P$ operate together and are in conflict. In the latter case, there is an interactive effect in the sense that the effect of $P$ on perceived height when cues conflict is larger than the isolated effect of $P$. A test for monotonic trend was used to test whether or not this interactive effect increased with increasing strength of the perspective cues. This trend was very significant $(\mathrm{z}=5.284)$.

\section{Relation Between Estimates of Depth and Height}

We found, in the foregoing, very significant trends in the depth and height judgments with increasing strength of the perspective cue. These trends in the height estimates were predicted by the size-distance invariance hypothesis, and this latter relation seems to hold for the conditions of this experiment. For every subject, the correlation between perceived rotation and perceived height was tested. Of the $18 \mathrm{sub}$ jects, 14 had a significant correlation and 4 did not. None of these 4 subjects, however, showed a tendency in the opposite direction.

In order to compare more directly the size of the effects of combining aniseikonic and perspective depth cues upon the judgments of depth and height, the distance of the left and right sides of the apparently rotated patterns from the position of the observer were calculated from the angles of rotation with a mean pupil distance of $64 \mathrm{~mm}$. These calculated distances were then expressed in percentages of the observation distance of $300 \mathrm{~cm}$. In Table 5 , these percentages are shown together with the estimates of height, expressed in percentages of the physical height.

The ratios of the percentages in Table 5 of perceived height to perceived depth are shown in Table 6. For the left side of the patterns, these ratios are nearly constant; for the right side of the patterns, this is the case only when aniseikonia and perspective cues are in conflict. When these cues operate in the same direction, the ratios increase with increasing strength of the perspective cue. It does not seem probable that this is caused by overestimation of the smaller right sides of the patterns, as this is not the 
Table 5

Perceived Distance of the Left and Right Sides of the Patterns Expressed in Proportion of the Observation Distance of $300 \mathrm{~cm}$, and Perceived Height of the Left and Right Sides of the Patterns Expressed in Proportion of the Physical Height

\begin{tabular}{|c|c|c|c|c|c|c|c|c|}
\hline \multirow{2}{*}{$\begin{array}{l}\text { Height of } \\
\text { Right Side }\end{array}$} & \multicolumn{2}{|c|}{$\overline{\mathrm{A}+\mathrm{P}}$ Left Side } & \multicolumn{2}{|c|}{$\overline{\Lambda+P}$ Right Side } & \multicolumn{2}{|c|}{$\overline{\mathbf{A}-\mathbf{P}}$ Left Side } & \multicolumn{2}{|c|}{$\overline{\mathrm{A}-\mathrm{P}}$ Right Side } \\
\hline & Depth & Height & Depth & Height & Depth & Height & Depth & Height \\
\hline 15.0 & 96.8 & 94.8 & 103.4 & 100.0 & 104.8 & 102.6 & 95.6 & 92.6 \\
\hline 12.0 & 96.1 & 93.9 & 104.3 & 102.0 & 103.6 & 101.2 & 96.6 & 94.2 \\
\hline 8.3 & 95.2 & 92.8 & 105.3 & 105.2 & 103.0 & 100.5 & 97.2 & 94.4 \\
\hline 5.0 & 94.1 & 92.7 & 106.6 & 108.3 & 102.4 & 100.0 & 97.7 & 95.1 \\
\hline 1.7 & 93.2 & 91.9 & 107.9 & 111.9 & 101.2 & 98.9 & 98.8 & 96.6 \\
\hline
\end{tabular}

Table 6

Height-Distance Ratios*

\begin{tabular}{llllrrr}
\hline & & \multicolumn{5}{c}{ Height of Right Side } \\
\cline { 3 - 7 } & & 15.0 & 12.0 & 8.3 & 5.0 & 1.7 \\
\hline Left & $\overline{\mathrm{A}+\mathrm{P}}$ & .979 & .977 & .975 & .985 & .986 \\
Side & $\overline{\mathrm{A}-\mathrm{P}}$ & .979 & .977 & .976 & .977 & .977 \\
Right & $\overline{\mathrm{A}+\mathrm{P}}$ & .967 & .978 & .999 & 1.016 & 1.037 \\
Side & $\overline{\mathrm{A}-\mathrm{P}}$ & .969 & .975 & .971 & .973 & .978 \\
\hline
\end{tabular}

*See text

case in either the conflict condition or the perspective condition.

The size-distance invariance hypothesis expressed as $S^{\prime} / D^{\prime}=k \theta$ holds for the conflict condition and for the left side of the condition of agreement. For the right side of the condition of agreement, the more general form, $S^{\prime} / D^{\prime}=k \theta^{n}$ (Gogel, 1971), seems to hold.

\section{Causal Analysis}

Simon (1954) presented causal models for threevariable cases. Blalock (1962) extended the use of partial correlations to the inference of causation in four-variable cases. A partial correlation is a net correlation between two variables when the influence of one or more additional variables has been eliminated from both. For instance, $r_{\text {Ad.P }}$ indicates a correlation between aniseikonia (A) and perceived depth (d) when the influence of perspective (P) has been eliminated from $\mathbf{A}$ and $\mathrm{d}$. This partial correlation becomes zero when the effect of $A$ on $d$ operates only through change of $\mathrm{P}$, whose influence has been eliminated in this correlation. By the use of partial correlation, we cannot decide the direction of causality. It is clear, however, that the causal arrows start from the stimulus variables $A$ and $P$ and not from the response variables $d$ and $h$.

According to Blalock, we have to compute firstorder and second-order partial correlations. Patterns of partial correlations having significant values suggest causal relations among variables. Blalock (1962) summarizes in series $A$, in which the experimental variables are independent, 16 models for which he gives the zero correlations and zero partial correlations. Oyama (1977) extended this list with derived zero partial correlations. We applied this type of analysis to our data.

Assuming linear relations between variables, the simple and partial correlations were calculated for the group means and for all subjects. The significant and insignificant simple and partial correlations are summarized in Table 7, with the exception of the correlations between $A$ and $P$, since the experimental model guarantees independence, for the group means and for seven individual subjects.

The analysis of the group means shows that $r_{P d}$ and $r_{P h}$ are insignificant. As the effect of $P$ on perceived depth and on perceived height in the agreement and in the conflict conditions is opposite, a nearly zero correlation may be expected here. The second-order partial correlations are significant, indicating a causal relation between perspective and perceived depth and between perspective and perceived height. The other second-order partial correlations are also significant, indicating causal relations between aniseikonia and perceived depth, between aniseikonia and perceived height, and between perceived depth and perceived height. This pattern of significant and insignificant correlations fits model A of Blalock's chart best. The causal relations indicated in model A are shown in Figure 2.

The results of seven subjects are also shown in Table 7. For Subjects 2 and 17, perspective dominated over aniseikonia. For both subjects, all secondorder partial correlations are significant, indicating causal relations between aniseikonia and perceived depth, aniseikonia and perceived height, perspective and perceived depth, perspective and perceived height, and perceived depth and perceived height. This pattern of significant second-order partial correlations fits model A of Blalock's chart best (Figure 2).

For the other five subjects from Table 7, aniseikonia dominated over perspective. For Subjects 5 , 9 , and 15, all second-order partial correlations are significant, so that model A (Figure 2) fits their outcomes best.

Subject 12 is one of the two subjects who suppressed the influence of perspective almost completely. Accordingly, the second-order partial correlations between perspective and perceived depth and between perspective and perceived height are not 
Table 7

Simple and Partial Correlations Between Aniseikonia (A), Perspective (P), Perceived Depth (d), and Perceived Height (h) for the Group Means and for Seven Individual Subjects

\begin{tabular}{|c|c|c|c|c|c|c|c|c|}
\hline & \multirow[b]{2}{*}{ Group } & \multicolumn{7}{|c|}{ Subjects } \\
\hline & & 5 & 6 & 9 & 12 & 2 & 17 & 15 \\
\hline $\mathrm{r}_{\mathbf{A d}}$ & $.919 * *$ & $.955 * *$ & $.959 * *$ & $.949 * *$ & $.970 * *$ & $.735^{* *}$ & .425 & $.974 * *$ \\
\hline $\mathrm{I}_{\mathrm{Ah}}$ & $.839 * *$ & $.897 * *$ & $.932 * *$ & $.895^{* *}$ & $.901 * *$ & .130 & .091 & $.962 * *$ \\
\hline $\mathrm{I}_{\mathbf{P d}}$ & .009 & .009 & .012 & .009 & .008 & .031 & .027 & -.020 \\
\hline $\mathrm{I}_{\mathbf{P h}}$ & .150 & .119 & .071 & .123 & .137 & $.549 *$ & $.458^{*}$ & .005 \\
\hline $\mathrm{I}_{\mathrm{dh}}$ & $.963 * *$ & $.974 * *$ & $.989 * *$ & $.976 * *$ & $.945^{* *}$ & $.497 *$ & $.830^{* *}$ & $.998 * *$ \\
\hline $\mathrm{r}_{\text {Ad.P }}$ & $.919 * *$ & $.955^{* *}$ & $.960 * *$ & $949 * *$ & $.970^{* *}$ & $.736 * *$ & .425 & $.974 * *$ \\
\hline red.h & $.751^{* *}$ & $.810^{* *}$ & $.702 * *$ & $.775^{* *}$ & $.833^{* *}$ & $.780^{* *}$ & $.630^{* *}$ & $.862 * *$ \\
\hline $\mathrm{r}_{\mathrm{Ah}, \mathrm{P}}$ & $.849 * *$ & $.903^{* *}$ & $.935^{* *}$ & $.902 * *$ & $.910^{* *}$ & .155 & .102 & $.962 * *$ \\
\hline $\mathrm{r}_{\text {Ah.d }}$ & -.418 & $-.516^{*}$ & -.406 & $-.455^{*}$ & -.191 & -.401 & $-.519^{*}$ & $-.792 * *$ \\
\hline $\mathrm{r}_{\mathrm{Pd} . \mathrm{A}}$ & .024 & .030 & .044 & .027 & .035 & .045 & .030 & -.090 \\
\hline $\mathrm{I}_{\mathrm{Pd} . \mathrm{h}}$ & .021 & $-.480^{*}$ & -.397 & $-.519 *$ & -.374 & -.334 & $-.713 * *$ & -.740 \\
\hline $\begin{array}{l}\text { IPh.A } \\
\text { Ph.n }\end{array}$ & .276 & .268 & .197 & .275 & .316 & $.554^{*}$ & $.460^{*}$ & .018 \\
\hline $\mathrm{r}_{\mathrm{Ph}, \mathrm{d}}$ & $.520^{*}$ & $.490^{*}$ & .402 & $.530^{*}$ & .394 & $.616^{* *}$ & $.781 * *$ & .084 \\
\hline $\mathrm{r}_{\mathrm{dh} . \mathrm{A}}$ & $.891^{* *}$ & $.901 * *$ & $.929 * *$ & $.902 * *$ & $.672^{* *}$ & $.598 * *$ & $.878^{* *}$ & $.922 * *$ \\
\hline $\mathrm{r}_{\mathrm{dh} . \mathrm{P}}$ & $.972 * *$ & $.981 * *$ & $.991 * *$ & $.983 * *$ & $.953^{* *}$ & $.575^{* *}$ & $.920 * *$ & $.999 * *$ \\
\hline${ }^{I_{\text {Ad.Ph }}}$ & $.754 * *$ & $.838 * *$ & $.697 * *$ & $.780 * *$ & $.817^{* *}$ & $.780^{* *}$ & $.851^{* *}$ & $.949 * *$ \\
\hline $\mathrm{I}_{\mathrm{Ah} . \mathrm{Pd}}$ & $-.476 *$ & $-.609 * *$ & -.425 & $-.520^{*}$ & -.193 & $-.483^{*}$ & $-.816 * *$ & $-.925 * *$ \\
\hline${ }^{\mathrm{r} P d . A h}$ & $-.511 *$ & $-.506^{*}$ & -.382 & $-.533^{*}$ & -.253 & $-.458^{*}$ & $-.880^{* *}$ & $-.830 * *$ \\
\hline IPh.Ad & $.563^{*}$ & $.555^{*}$ & .421 & $.582 * *$ & .396 & $.658^{* *}$ & $.901 * *$ & $.827 * *$ \\
\hline $\mathrm{r}_{\mathrm{dh} . \mathrm{AP}}$ & $.921 * *$ & $.927 * *$ & $.939 * *$ & $.931 * *$ & $.697 * *$ & $.689 * *$ & $.974 * *$ & $.997^{* *}$ \\
\hline
\end{tabular}

significant. Only $r_{\text {Ad.Ph }}$ and $r_{d h . A P}$ are significant, indicating causal relations between aniseikonia and perceived depth and between perceived depth and perceived height. Blalock's chart does not give models for causal relations of less than three arrows. In Figure 2, the causal relations are shown in model A-X.

For Subject 6, only $r_{\text {Ad.Ph }}$ and $r_{d h . A P}$ are significant. The other second-order partial correlations do not reach the critical value of .466 . Model A-X (Figure 2) fits this outcome best.

For all 18 subjects, a significant second-order partial correlation between perceived depth and perceived height was found. Twelve subjects showed patterns of significant second-order partial correlations in accordance with model $A$, four subjects showed patterns in accordance with model A-X; for two subjects, only the second-order partial correlation between perceived depth and perceived height was significant.

From this analysis, we can conclude that for most subjects perceived depth is directly determined by aniseikonia and perspective cues, and perceived height is directly determined by aniseikonia and perspective cues and indirectly through perceived depth. Four

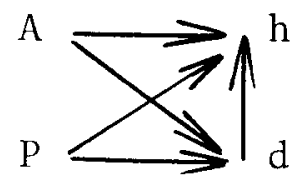

Mode1 A

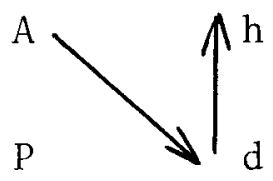

Mode1 A-X
Figure 2. The causal models from the causal analysis. subjects more or less suppressed the effect of perspective, and 2 subjects were inconsistent observers, having only a significant second-order partial correlation between perceived depth and perceived height. For all 18 subjects, a causal relation between perceived depth and perceived height was found.

\section{DISCUSSION AND CONCLUSION}

We found that for simple geometric patterns, as used in this experiment, both binocular disparity and perspective cues affect perceived depth and perceived height. For 12 subjects, binocular disparity dominated over perspective, and for 6 subjects, perspective dominated over binocular disparity.

For the judgment of depth, the effects of binocular disparity and of perspective cues were additive. This was the case both when these depth cues operated in the same direction and when they were in conflict.

The effects of binocular disparity and perspective cues on perceived height showed some interaction in the sense that when both operated in the same direction their combined effect was stronger than the sum of the separate effects. When binocular disparity and perspective cues were in conflict, their combined effect was less than the difference of the separate effects of binocular disparity and perspective cues. The effect of the perspective cues operating together with binocular disparity, both in the same and in the opposite direction, on perceived height, seems to be stronger than the effect of the perspective cues operating separately. That this interactive effect is especially due to the perspective cue is obvious from 
the fact that the interactive effect increases with increasing strength of the perspective cue, while binocular disparity remains constant in all conditions. This trend was found to be very significant.

The size-distance invariance hypothesis holds at least in its general form under the conditions of this experiment. In the introduction, we mentioned at least three cases in which this invariant relationship can be explained: (1) The effects of the stimulus variables $A$ and $P$ operate directly on perceived depth, and they operate on perceived height only through change of perceived depth. (2) The effects of the stimulus variables $A$ and $P$ operate directly on perceived height, and they operate on perceived depth only through change of perceived height. (3) The effects of A and P on both perceived depth and perceived height operate directly and independently. Perceived depth and perceived height are mutually related only through their common determinants, $A$ and $P$. In the analysis of our data, we found a mixture of Explanation 1 and Explanation 3: Perceived depth was directly determined by $A$ and $P$, but perceived height was directly determined by $\mathbf{A}$ and $P$ and indirectly through change of perceived depth. There was a direct causal relation between perceived depth and perceived height.

Mori and Watase (cited by Oyama, 1977), using visual angle and physical distance as stimulus variables, found also a direct causal relation between perceived size and perceived distance for most of their subjects. On the other hand, Oyama (1974), using visual angle and convergence as stimulus variables, found, except in the case of one of his six subjects, no relation between perceived size and perceived distance. Both were directly and independently determined by the stimulus variables. It seems, therefore, that whether or not a direct causal relation between perceived depth and perceived height will be found depends on the stimulus variables and on the way subjects use information from the stimulus variables.

In the present study, we found a direct causal relation between the stimulus variables and the response variables, and a direct causal relation between perceived depth and perceived height. This suggests that the subjects also used the information from the judged depth to determine the perceived height of the sides of the patterns. This is consistent with our preceding suggestion from the analysis concerning additivity of the effects of aniseikonia and perspective cues on perceived height, namely, that the effects of perspective cues operating together with aniseikonia are stronger than the effects of perspective operating separately. When the patterns are seen in a frontoparallel plane, the effect of perspective cues is less than when these patterns are apparently rotated.

\section{REFERENCE NOTE}

1. Meer, H. C. van der. Stereoscopic depth perception under conditions of vertical aniseikonia: The influence of some stimulus variables. Report No. 71-HB-72-Ex, University of Groningen, 1971.

\section{REFERENCES}

Blalock, H. M., Four-variable causal models and partial correlations. American Journal of Sociology, 1962, 68, 182-194.

Epstein, W., \& Landauer, A. A. Size and distance judgments under reduced conditions of viewing. Perception \& Psychophysics, 1969, 6, 269-272.

Epstein, W., \& Morgan-Paap, C. L. The effect of level of depth processing and degree of informational discrepancy on adaption to uniocular image magnification. Journal of Experimental Psychology, 1974, 102, 585-594.

Gillam, B. J. Perception of slant when perspective and stereopsis conflict. Journal of Experimental Psychology, 1968, 78, 299-305.

GOGEL, W. C. The validity of the size-distance invariance hypothesis with cue reduction. Perception \& Psychophysics, $1971,9,92-94$.

HARKER, G. S. Interrelation of monocular and binocular acuities in the making of equidistance judgment. Journal of the Optical Society of America, 1958, 48, 233-240.

JAmESON, D., \& HuRvich, L. M. Note on factors influencing the relation between stereoscopic acuity and observation distance. Journal of the Optical Society of America, 1959, 49, 639.

Landauer, A. A. \& Epstein, W. Does retinal size have a unique correlate in perceived size? Perception \& Psychophysics, $1969,6,273-275$.

MeER, H. C. VAN DER. Linear combinations of stereoscopic depth effects in dichoptic perception of gratings. Vision Research, $1978,18,707-714$.

OgLe, K. N. Researches in binocular vision. New York: Hafner, 1964.

Oyama, T. Perceived size and perceived distance in stereoscopic vision and an analysis of their causal relations. Perception \& Psychophysics, 1974, 16, 175-181.

Oyama, T. Analysis of causal relations in the perceptual constancies. In W. Epstein (Ed.), Stability and constancy in visual perception. New York: Wiley, 1977.

Schriever, W. Experimentelle Studien über stereoskopisches Sehen. Zeitschrift für Psychologie, 1925, 96, 113-170.

Simon, H. A. Spurious correlation: A causal interpretation. Journal of the American Statistical Association, 1954, 49, 467479. (Chapter 3 in H. A. Simon, Models of man. New York: Wiley, 1957.)

\section{NOTE}

1. For most subjects, the degree of rotation was larger when the image of the left eye was magnified than when the image of the right eye was magnified. Ogle (1964) attended to this phenomenon. The differences found in this experiment are, however, somewhat larger than usually found. Van der Meer (1978) found a relatively large difference between the rotations in case of magnification of the images of the left and the right eye with spatial frequencies of 5 cycles/deg and lower, while the difference leveled off at higher spatial frequencies.

(Received for publication March 1, 1979; revision accepted September 28, 1979.) 